\title{
A!
}

This is an electronic reprint of the original article.

This reprint may differ from the original in pagination and typographic detail.

Laakkonen, J.; Nieminen, R. M.

\section{Resistivity of Ca-Al metallic glasses}

Published in:

Physical Review B

DOI:

10.1103/PhysRevB.34.567

Published: 15/07/1986

Document Version

Publisher's PDF, also known as Version of record

Please cite the original version:

Laakkonen, J., \& Nieminen, R. M. (1986). Resistivity of Ca-Al metallic glasses. Physical Review B, 34(2), 567577. https://doi.org/10.1103/PhysRevB.34.567

This material is protected by copyright and other intellectual property rights, and duplication or sale of all or part of any of the repository collections is not permitted, except that material may be duplicated by you for your research use or educational purposes in electronic or print form. You must obtain permission for any other use. Electronic or print copies may not be offered, whether for sale or otherwise to anyone who is not an authorised user. 


\title{
Resistivity of Ca-Al metallic glasses
}

\author{
J. Laakkonen and R. M. Nieminen* \\ Laboratory of Physics, Helsinki University of Technology, SF-02150 Espoo 15, Finland
}

(Received 11 December 1985)

\begin{abstract}
The resistivity of $\mathrm{Ca}-\mathrm{Al}$ metallic glass is calculated as a function of temperature and composition. The diffraction model is used, including the partial dynamic structure factors. To account for the blurring of the Fermi surface we propose a new model which preserves the wave-packet character of the scattering electron. The results compare favorably with the experimental measurements available, indicating that the diffraction model is applicable also for high-resistivity systems. The use of $t$-matrix formulation of scattering is seen essential and the value of the Fermi wave vector to be used is shown to need careful consideration. The Fermi-surface blurring is found to affect the resistivity but the changes are not drastic. The origins of the large resistivity are seen to be in the strong scattering from $\mathrm{Ca}$ atoms, as expected, but also in the changes in the atomic and electronic structure.
\end{abstract}

\section{INTRODUCTION}

In an earlier publication ${ }^{1}$ (hereafter referred to as paper I) we reported calculations of the resistivity and thermopower of $\mathrm{Mg}-\mathrm{Zn}$ metallic glasses. We applied the diffraction model using dynamical partial structure factors and also considered the effect of the Fermi-surface blurring due to the finite mean free path of electrons. Except for the composition dependence of the thermopower, the model was found to describe well the electric transport properties of amorphous $\mathrm{Mg}-\mathrm{Zn}$ alloys. Here we further improve the method by taking into account the effect of blurring the Fermi surface more rigorously and by using the $t$-matrix formulation for the electron-ion interaction instead of a simple model potential. In addition to blurring the Fermi surface the finite electron mean free path also has an effect on the electron-phonon interaction. This has been discussed extensively by Cote and Meisel ${ }^{2,3}$ and their treatment is adapted here. Finally, a minor change from paper $I$ is to be found also in the treatment of the multiphonon terms of structure factors. This framework is then applied to a detailed investigation of $\mathrm{Ca}-\mathrm{Al}$ amorphous alloys.

Previously, ${ }^{1}$ the effect of blurring of the Fermi surface due to the finite electron mean free path was included as suggested by Ferraz and March. ${ }^{4}$ In this method, basically, the free-electron density matrix is substituted for by a product of a free-electron density matrix and an exponentially decaying factor which describes the decay of correlations due to scattering. This leads to a resistivity formula with an integral over momentum-transfer vector $q$ from zero to infinity. This formulation apparently does not conserve momentum for $q>2 k_{F}$ ( $k_{F}$ is the Fermi wave vector). Furthermore, the probability of existence of electrons with a wave vector considerably greater than $k_{F}$ is highly improbable as given by the Fermi-Dirac distribution. This is not accounted for by the method of Ferraz and March. For this reason we choose a new approach and consider the scattering problem in terms of wave packets.
In the diffraction model the electron-ion interaction has traditionally been described by a (weak) pseudopotential. Here, instead, we apply the $t$-matrix formulation. This we do to eliminate the choice between different pseudopotentials and to be able to describe accurately scattering from one scatterer. The latter argument is important especially when considering high-resistivity systems. For comparison, however, we give some results computed using the Ashcroft model potential and an $a b$ initio pseudopotential.

When compared with the $\mathrm{Mg}-\mathrm{Zn}$ alloy discussed in paper I, the Ca-Al metallic glass is an interesting case for several reasons. Firstly, even though calcium and aluminum are simple metals (like $\mathrm{Mg}$ and $\mathrm{Zn}$ ), the resistivities measured for the Ca-Al alloys are extremely high $(\rho \geq 300$ $\mu \Omega \mathrm{cm}$ ) and depend strongly on the composition of the alloy. ${ }^{5}$ For $\mathrm{Mg}-\mathrm{Zn}$ alloys the measured resistivities are well below $100 \mu \Omega \mathrm{cm}$. This is usually considered a condition for the applicability of the diffraction model. It will thus be of interest to see how well the diffraction model works in this intermediate- or strong-scattering case. The aim is thus to carry the refined diffraction model through as completely as possible. Comparison with experiment then reveals the importance of other possible localization mechanisms and of effects beyond the present treatment, such as charge transfer in the heterovalent system.

The paper is organized as follows: Sec. II contains the formulation of the resistivity calculation, the results of which are presented in Sec. III. Section IV contains a discussion and conclusions.

\section{CALCULATION OF THE RESISTIVITY}

The high electrical resistivity of metallic glasses implies that the long electronic mean free path of crystalline metals is severely diminished in the amorphous state. An electron can no longer be described as a delocalized (plane) wave with sharp wave-vector value $k$, but one has to deal with wave packets and a spread of $k$ values. This certainly is important in such cases as Anderson localization, but 
it may have a considerable effect on the resistivity even well before the onset of localization.

To see easily how the wave-packet formulation enters the diffraction model, it is best to go to the original Ziman derivation ${ }^{6}$ of the model. The resistivity $\rho$ is computed from the differential scattering cross section $d \sigma(\theta) / d \Omega$ as

$$
\rho \propto \int_{0}^{2 \pi}(1-\cos \theta) \frac{d \sigma}{d \Omega} \sin \theta d \theta .
$$

A formula for $d \sigma / d \Omega$ is derived in standard quantummechanics texts ${ }^{7}$ by considering a wave packet,

$$
\psi(\mathbf{r}, 0)=\frac{1}{(2 \pi)^{3 / 2}} \int d^{3} k \varphi(\mathbf{k}) e^{i \mathbf{k} \cdot\left(\mathbf{r}-\mathbf{r}_{0}\right)},
$$

where the wave packet is defined by a smooth function $\varphi$ of width $\Delta \mathbf{k}$ and centered around a mean momentum $\mathbf{k}_{0}$. $\mathbf{r}_{0}$ is the position of the packet at time $t=0$. It is usually assumed that $\varphi$ is a peaked function and that the scattering amplitude $f_{\mathbf{k}_{0}}(\theta)$ is a slowly varying function over the range where $\varphi$ is appreciably different from zero (near $\mathbf{k} \simeq \mathbf{k}_{0}$ ). Then one obtains

$$
\frac{d \sigma}{d \Omega}=\left|f_{\mathbf{k}_{0}}(\theta)\right|^{2} \text {. }
$$

When $\left|f_{\mathbf{k}_{0}}(\theta)\right|^{2}$ is now expanded using either the Born approximation or the partial-wave series, the regular forms of the diffraction-model equations for the resistivity are recovered. While this assumption is valid in most cases, in some metallic glasses the resistivity $\rho$ may be so large that the electronic mean free path $l$ as computed from the Drude formula (atomic units are used here and in the following),

$$
\rho \cong k_{F} / n_{e} l,
$$

is of the order of one or two lattice distances. Then the uncertainty in the position of the electron is $\Delta x \sim l$, and from the uncertainty principle $\Delta x \Delta k \geq 1$ one obtains

$$
\Delta k \approx s / l \text {, }
$$

where $s$ is a number of the order of 1 . Since typically $k_{F} \gtrsim 0.6$ a.u., one sees that the width of the wave packet can be a sizable fraction of the mean momentum of the wave packet $\left(k_{0} \simeq k_{F}\right)$ and the approximation (3) may break down. When this assumption is relaxed, one obtains, instead of Eq. (3) (for details see the Appendix),

$$
\frac{d \sigma}{d \Omega}=\frac{\int d^{3} k|\varphi(\mathbf{k})|^{2}\left|f_{\mathbf{k}}(\theta)\right|^{2}}{\int d^{3} k|\varphi(\mathbf{k})|^{2}} .
$$

For the shape $\varphi(\mathbf{k})$ of the wave packet, below we use both Gaussian and Lorentzian forms. For the Gaussian the wave packet

$$
\varphi(\mathbf{k})=\exp \left[-\left(\mathbf{k}-\mathbf{k}_{0}\right)^{2} / 2(\Delta k)^{2}\right],
$$

and for the Lorentzian,

$$
\varphi(\mathbf{k})=\frac{1}{\left(\left|\mathbf{k}-\mathbf{k}_{0}\right|+i \Delta k\right)^{n}},
$$

where $n$ is an integer.

To compute the energy-dependent phase shifts in the partial-wave expression for $f_{k}(\theta)$, one needs a potential $V(r)$. This we take to be the atomic potential of the particular atom ( $\mathrm{Ca}$ or $\mathrm{Al})$ calculated using the HermanSkillman ${ }^{8}$ programs, shifted so that the potential vanishes at the Wigner-Seitz-cell boundary (assumed spherical). Thus, with this "crystal zero,"

$$
V(r)=V_{\mathrm{at}}(r)-V_{\mathrm{at}}(R),
$$

where $V_{\text {at }}$ is the atomic potential and

$$
R=\left(\frac{3}{4 \pi n}\right)^{1 / 3}
$$

with $n$ equal to the atomic density of the alloy. Other effects due to the condensed phase were neglected.

For comparison, the use of a pseudopotential for the matrix element instead of the partial-wave expansion was studied. Two potentials, the Ashcroft model potential ${ }^{9}$ and an $a b$ initio pseudopotential, ${ }^{10}$ were considered. The empty core radius $\boldsymbol{R}_{\boldsymbol{c}}$ of the Ashcroft model potential was determined as in paper I, i.e., by fitting the liquid-metal resistivities. Using typical values for the packing fraction $\eta$ and the hard-sphere diameter $\sigma$ of liquid metals, ${ }^{11}$ we obtain $R_{c}(\mathrm{Al})=0.624 \AA$ and $R_{c}(\mathrm{Ca})=0.910 \AA$. No correction for the possible charge-transfer effects was applied. The $a b$ initio pseudopotential parameters were taken from Ref. 10. The screening was included by using the dielectric function given by Taylor. ${ }^{12}$

Cote and $\mathrm{Meisel}^{2,3}$ have noted that the change in the electron-phonon interaction due to the finite electron mean free path can be taken into account through the Pippard-Ziman condition. ${ }^{13}$ In short, this means that if the phonon wavelength exceeds the electron mean free path $l$, the electron does not "see" the phonon and the scattering is diminished. In the calculations this is includ$\mathrm{ed}^{2,3}$ by multiplying the one-, two-, etc. phonon terms of the dynamical structure factor appearing in the phonon wave-number integral by the Pippard function ${ }^{13}[F(q l)]$ :

$$
F(y)=\frac{2}{\pi}\left(\frac{y \tanh ^{-1} y}{y-\tanh ^{-1} y}-\frac{3}{y}\right) .
$$

With these refinements the resistivity $\rho$ can now be computed from the equation (also see paper I)

$$
\begin{aligned}
\rho= & \frac{n_{i}}{3 \pi n_{e}^{2}} \frac{1}{\int d^{3} k|\varphi(k)|^{2}} \\
& \times\left.\int d^{3} k \frac{1}{k^{2}}|\varphi(k)|^{2} \int_{0}^{2 k} d q q^{3} \int_{-\infty}^{\infty} \frac{d \omega}{2 \pi} \frac{\beta \omega}{\exp (\beta \omega)-1}\left|c_{a}\right| \sum_{m=0}^{\infty}(2 m+1) e^{i \delta_{m}^{a}} \sin \delta_{m}^{a} P_{m}(\cos \theta)\right|^{2} S_{a a}(q, \omega)
\end{aligned}
$$




$$
+c_{b}\left|\sum_{m=0}^{\infty}(2 m+1) e^{i \delta_{m}^{b}} \sin \delta_{m}^{b} P_{m}(\cos \theta)\right|^{2} S_{b b}(q, \omega)+\left(c_{a} c_{b}\right)^{1 / 2} \mid \sum_{m} \sum_{n}(2 m+1)(2 n+1) \sin \delta_{m}^{a}
$$

$$
\begin{aligned}
& \times \sin \delta_{n}^{b} P_{m}(\cos \theta) P_{n}(\cos \theta) \\
& \times\left(e^{i\left(\delta_{m}^{a}-\delta_{n}^{b}\right)}+e^{i\left(\delta_{n}^{b}-\delta_{m}^{a}\right)}\right)\left|S_{a b}(q, \omega)\right|,
\end{aligned}
$$

where

$$
\cos \theta=1-q^{2} / 2 k^{2} \text { and } n_{e}=k_{F}^{3} / 3 \pi^{2} .
$$

Above, $n_{i}$ and $n_{e}$ are the ion and electron densities, respectively, $c_{a}$ and $c_{b}$ are the concentrations $\left[c_{a}=N_{a} /\left(N_{a}+N_{b}\right)\right.$, etc.] of species $a$ and $b, \delta_{m}^{a}$ and $\delta_{m}^{b}$ are the $m$ th phase shifts corresponding to the potentials of atoms of type $a$ and $b, \beta=1 / k_{B} T$, and $S_{a b}(k, \omega)$ is the dynamical partial structure factor. For the shape of the wave packet $[\varphi(k)]$, the functions of Eqs. (7) and (8) are used and the $k$ integrals are over the whole $\mathbf{k}$ space. In (7) and (8), $k_{0}$ denotes the mean momentum of the wave packet (i.e., the momentum of the scattering electron) for which the Fermi wave vector $k_{F}$ was used. The wave vector $\mathbf{q}$ is the momentum transfer between the Fourier components of the scattering wave packet, and the limits of the $q$ integral (from 0 to $2 k$ ) ensure the conservation of momentum. The electron mean free path $l$ is determined self-consistently using Eq. (4). When computing the width of the wave packet $\Delta k$, the relationship $\Delta k \sim 1 / l$ is reasonable even if the exact value of the parameter $s$ in Eq. (5) is not known. Therefore, we first computed the resistivity as a function of $s$ and then fixed $s$ for different types of wave packet. The order for the Lorentzian-type wave packet [power $n$ in Eq. (8)] was chosen to be $n=10$. The phase shifts in (12) were included up to angularmomentum quantum number $l=10$.

The dynamical partial structure factor was computed in the same way as in paper I, except for one change. The multiphonon contribution is now taken into account by using the Sham-Ziman approximation, ${ }^{14}$ i.e., inclusion of the multiphonon terms is assumed to cancel the DebyeWaller factor in the one-phonon term.

Next, the parameters of the model are given. For the details of the model we refer to paper I. The density $d$ of the $\mathrm{Ca}-\mathrm{Al}$ alloy was determined from a graph fitted to the values measured ${ }^{5}$ for $\mathrm{Ca}_{80} \mathrm{Al}_{20}$ and $\mathrm{Ca}_{60} \mathrm{Al}_{40}$ metallic glasses and those of pure $\mathrm{Ca}$ and $\mathrm{Al}$ (crystalline) metals: For $\mathrm{Ca}_{80} \mathrm{Al}_{20}, d=1.714 \mathrm{~g} / \mathrm{cm}^{3}$; for $\mathrm{Ca}_{60} \mathrm{Al}_{40}, d=1.936$ $\mathrm{g} / \mathrm{cm}^{3}$; for $\mathrm{Ca}, d=1.54 \mathrm{~g} / \mathrm{cm}^{3}$; and for $\mathrm{Al}, d=2.70$ $\mathrm{g} / \mathrm{cm}^{3}$. The Wigner-Seitz radius $R$ was fixed to the value of 3.64 a.u. The hard-sphere diameters $\sigma_{\mathrm{Ca}}$ and $\sigma_{\mathrm{Al}}$ were obtained from the nearest-neighbor distances calculated by Hafner ${ }^{15}$ for the amorphous $\mathrm{Ca}_{50} \mathrm{Al}_{50}$ compound. In order not to exceed the fcc maximum packing density for any composition, we slightly decreased the values given by
Hafner (keeping the ratio $\sigma_{\mathrm{Ca}} / \sigma_{\mathrm{Al}}$ constant) and used the following diameters: $\sigma_{\mathrm{Ca}}=3.41 \AA$ and $\sigma_{\mathrm{Al}}=2.86 \AA$.

The sound velocities were determined as in paper I, i.e., by scaling from the pure-metal values by $1 / \sqrt{d}$. The longitudinal and transverse speeds of sound used are, for $\mathrm{Al},{ }^{16} v_{\mathrm{L}}=6300 \mathrm{~m} / \mathrm{s}$ and $v_{\mathrm{T}}=3230 \mathrm{~m} / \mathrm{s}$, and, for $\mathrm{Ca},{ }^{17}$ $v_{\mathrm{L}}=4030 \mathrm{~m} / \mathrm{s}$ and $v_{\mathrm{T}}=2190 \mathrm{~m} / \mathrm{s}$. In the glassy metal the same value for the longitudinal speed of sound was used as for the crystalline material; the transverse speed was decreased by $15 \%$ from the corresponding crystalline value. ${ }^{18}$

An important parameter is the Fermi wave vector $k_{F}$. Previously, ${ }^{1}$ it was determined directly by the freeelectron formula, but in the case of Ca-Al alloy this method is obviously not as justified as for $\mathrm{Mg}-\mathrm{Zn}$. This is indicated by the apparent $k_{F}$ value determined from the Hall-effect measurements: According to these measurements, $k_{F}$ either stays nearly constant ${ }^{19}$ or changes with composition, ${ }^{20}$ being considerably smaller than the freeelectron value. Following the former experimental result we used in our calculations, $k_{F}=1.18 \AA^{-1}$ for the whole composition range. For comparison, some calculations were repeated with $k_{F}$ values determined directly from the free-electron formula.

\section{RESULTS}

Figure 1 shows the calculated four largest scattering phase shifts as a function of energy (as measured from the crystal zero) for $\mathrm{Al}$ and $\mathrm{Ca}$. From Fig. 1(a) one sees that, for $\mathrm{Al}, s$ and $p$ scattering dominate and the $d$ contribution emerges gradually only at high energies. For $\mathrm{Ca}$ [Fig. 1 (b)] the situation is different: the $d$-phase shift changes now abruptly at $k=1.2 \AA^{-1}$, resembling a resonance. This results in strong $d$-wave scattering. Band-structure calculations for $\mathrm{Ca}-\mathrm{Al}$ metallic glass ${ }^{21}$ also show the significance of Ca-derived $d$ states.

When studying the effect of a finite electron mean free path using the framework presented in the preceding section, one has to specify the width of the wave packet $\Delta k$ via Eq. (5). Obviously, one cannot unambiguously determine $s$ and therefore the resistivity of two $\mathrm{Ca}-\mathrm{Al}$ metallic glass compounds at $T=300 \mathrm{~K}$ were computed as a function of $s$. The results for $\mathrm{Ca}_{60} \mathrm{Al}_{40}$ shown in Fig. 2(a) indicate a common behavior for both types of wave packets: With increasing $s$ the resistivity at first decreases by about 

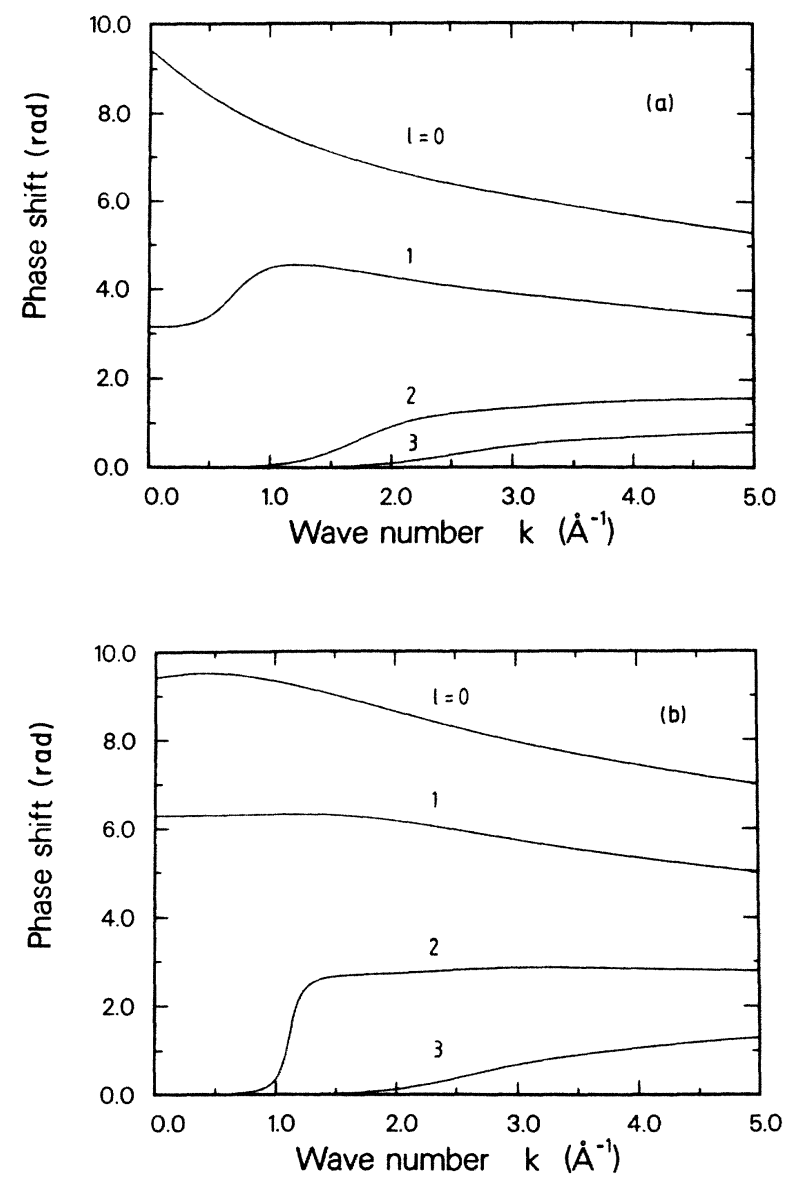

FIG. 1. Phase shifts for $l=0,1,2,3$ as a function of energy for (a) $\mathrm{Al}$ and (b) $\mathrm{Ca}$. The energy is measured from the crystal zero, which is $5.925 \mathrm{eV}$ below the zero of energy for $\mathrm{Al}$ and $7.201 \mathrm{eV}$ for $\mathrm{Ca}$.

$30 \%$ from the value at $s=0$, and then starts to increase until a divergence sets in. The limiting value for $s \rightarrow 0$ corresponds to $\Delta k \rightarrow 0$, i.e., a sharp Fermi surface. In this limit the resistivities computed using different forms for the wave packet converge on each other and the regular, sharp Fermi surface results. Large $s$ values mean, on the other hand, a wide packet. Then the uncertainty principle allows a small uncertainty for the particle position, i.e., the particle becomes localized and the resistivity increases sharply. The behavior of the resistivity for intermediate $s$ values is interesting: it appears that in the case of $\mathrm{Ca}_{60} \mathrm{Al}_{40}$, a wave packet having a small finite width encounters a smaller resistivity and can thus more easily propagate through the sample than a pure plane wave. Furthermore, the detailed form of the wave packet does not seem to be important and the decrease of the resistivity is about the same for both types. Slightly different positions for the minimum and the divergence are expected since, e.g., the full width at half maximum (FWHM) of the wave packet has a different relationship to $\Delta k$ for the two wave packets. The existence of a resistivity minimum for a wave packet, however, is not a general property. This is shown by Fig. 2(b), where the results for a
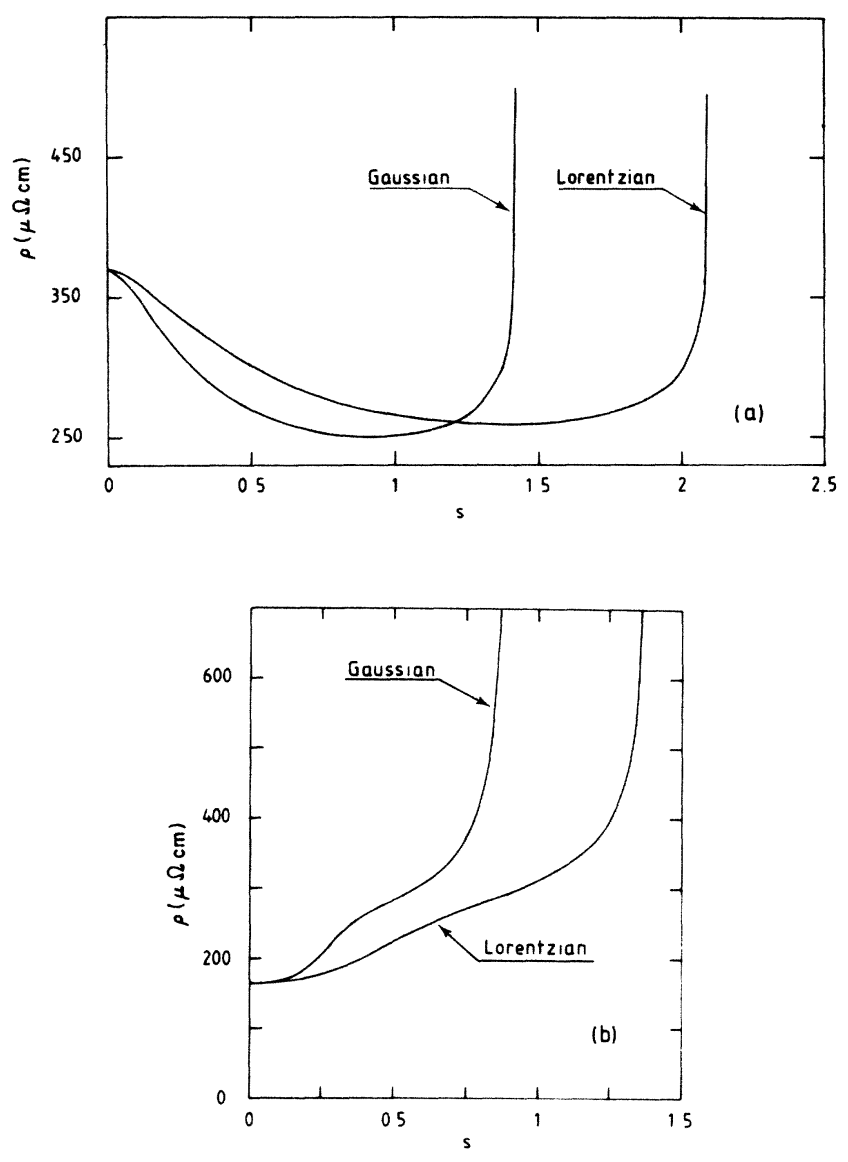

FIG. 2. Resistivity of (a) $\mathrm{Ca}_{60} \mathrm{Al}_{40}$ and (b) $\mathrm{Ca}_{30} \mathrm{Al}_{70}$ metallic glasses at $T=300 \mathrm{~K}$ as a function of the wave-packet-width parameter $s$. Two forms for a wave packet are considered: Gaussian and Lorentzian.

$\mathrm{Ca}_{30} \mathrm{Al}_{70}$ compound are given. Now the resistivity increases continuously with increasing wave-packet width.

When choosing a value for the parameter $s$ in the case in which a resistivity minimum exists, one should obviously stay with $s$ values less than or equal to the $s$ value that yields the resistivity minimum [see Fig. 2(a)]; if the minimum is passed, the wave packet spreads too much and a divergence results. Therefore, to study the effect of blurring the Fermi surface in the case of $\mathrm{Ca}_{60} \mathrm{Al}_{40}$, two values of $s$ were used for both wave packets: one that yields about a $15 \%$ decrease in the resistivity from the sharp Fermi-surface value, and another that corresponds to the minimum of that curve (30\% decrease). These values for the Gaussian wave packet are $s=0.25$ and 0.93 , and for the Lorentzian wave packet, $s=0.40$ and 1.38. Then the corresponding FWHM values of the wave packets are (for $\mathrm{Ca}_{60} \mathrm{Al}_{40}$ ), for the Gaussian packet, 0.20 and $0.62 \AA^{-1}$, and, in the case of the Lorentzian packet, 0.11 and $0.32 \AA^{-1}$. These are rather sizable fractions of the mean momentum of the wave packet, i.e., $k_{F}(=1.18$ $\AA^{-1}$ ). If the resistivity increases continuously with the wave-packet width [see Fig. 2(b)], then one just has to stay away from the regions where the divergent behavior sets 
in. To study the effect of Fermi-surface blurring for the $\mathrm{Ca}_{30} \mathrm{Al}_{70}$ compound, the values $s=0.25$ and 0.5 were used.

The temperature dependence of the resistivity of the $\mathrm{Ca}_{60} \mathrm{Al}_{40}$ compound is presented in Fig. 3. When compared with the experimental results ${ }^{5,20}$ one sees that the sharp Fermi-surface curve with $k_{F}=1.18 \AA^{-1}$ gives the best fit: Experimentally, the $\rho(T)$ curve decreases monotonously with increasing temperature, approximately parabolically at lowest temperatures, and linearly at higher temperatures. In Fig. 3 this is qualitatively reproduced well, except for a small discrepancy below $50 \mathrm{~K}$. Quantitatively, the change in $\rho(T)$ values between $T=0$ and 300 $\mathrm{K}$ is theoretically considerably less than the experimental findings $(2-3 \%$ versus $11-13 \%)$. We shall return to this question in the following section. The experimental room-temperature value for the resistivity (381-433 $\mu \Omega \mathrm{cm}$ ), however, is well reproduced theoretically (see Fig. 2). Figure 3 clearly shows how the inclusion of the effect of a blurred Fermi surface does not drastically change the form of the temperature dependence even when a very wide wave packet is used: with increasing $s$ the temperature dependence only becomes gradually weaker. Once again, no essential differences between the two wavepacket forms can be seen. In addition to the Fermiwave-vector value $k_{F}=1.18 \AA^{-1}$, the temperature dependence for the sharp Fermi was also calculated using the $k_{F}$ value given by the free-electron formula $\left(k_{F}=1.33\right.$ $\AA^{-1}$ ). In this case the change is more pronounced: The

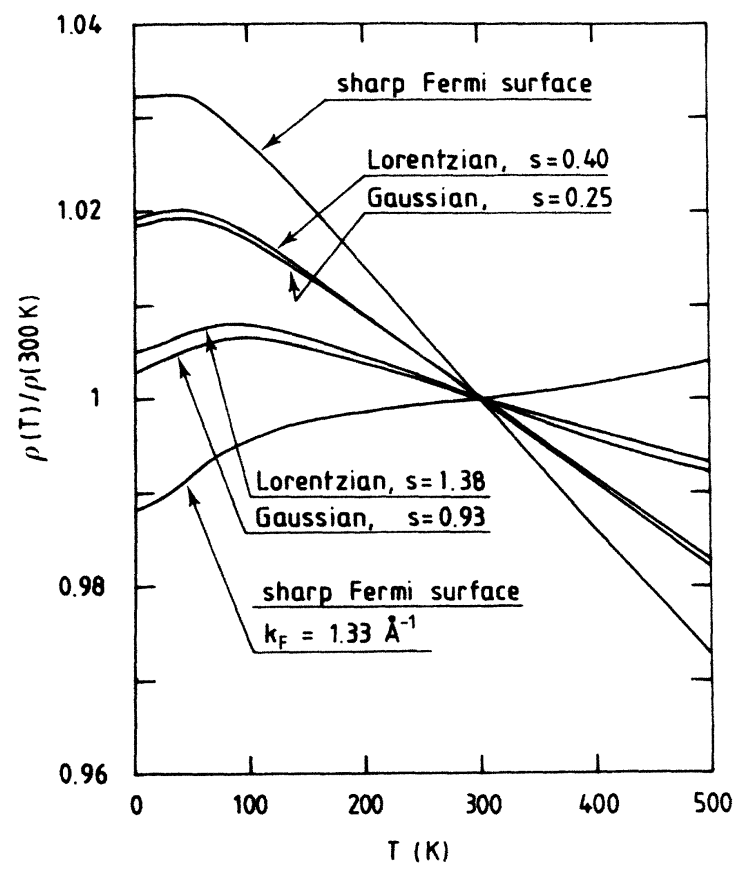

FIG. 3. Resistivity of $\mathrm{Ca}_{60} \mathrm{Al}_{40}$ metallic glass as a function of temperature. Two of the curves are computed using the sharpFermi-surface formula, while the others take into account the Fermi-surface blurring. The type of wave packet and the value of the width parameter $s$ are shown. If not otherwise indicated, the value of the Fermi wave vector used is $k_{F}=1.18 \AA^{-1}$. temperature coefficient of resistivity (TCR) becomes positive and the magnitude of resistivity at $T=300 \mathrm{~K}$ drops from 369 to $128 \mu \Omega \mathrm{cm}$.

Figure 4 shows the resistivity as a function of temperature for the $\mathrm{Ca}_{30} \mathrm{Al}_{70}$ amorphous alloy. Now the curve corresponding to a sharp Fermi surface (i.e., $s=0$ ) is seen to give a resistivity that increases monotonously with temperature, i.e., the TCR, defined as $\alpha=(1 / \rho)(d \rho / d T)$, is positive. In this case the inclusion of the Fermi-surface blurring decreases the room-temperature value of the TCR and makes the temperature dependence steeper when the width of the wave packet increases.

According to these results, the wave-packet formulation has different effects on the temperature dependence of resistivity for the $\mathrm{Ca}_{60} \mathrm{Al}_{40}$ and $\mathrm{Ca}_{30} \mathrm{Al}_{70}$ systems: In the former case the TCR increased and in the latter case it decreased when the wave-packet width increased. To study this apparently contradictory behavior, we have plotted, in Fig. 5, the TCR as a function of resistivity at room temperature. Except for one point (the $\mathrm{Ca}_{30} \mathrm{Al}_{70}$ alloy with the widest Gaussian wave packet), the dependence of the TCR on resistivity is seen to be linear in both cases. This kind of behavior is also well known experimentally when the TCR's of different compositions are studied (the socalled Mooij correlation). ${ }^{22}$ Now it is obvious that the inclusion of Fermi-surface blurring either increases or decreases (nearly linearly) the room-temperature TCR, depending on whether the resistivity is decreased or increased, respectively. The qualitative form of the $\rho(T)$

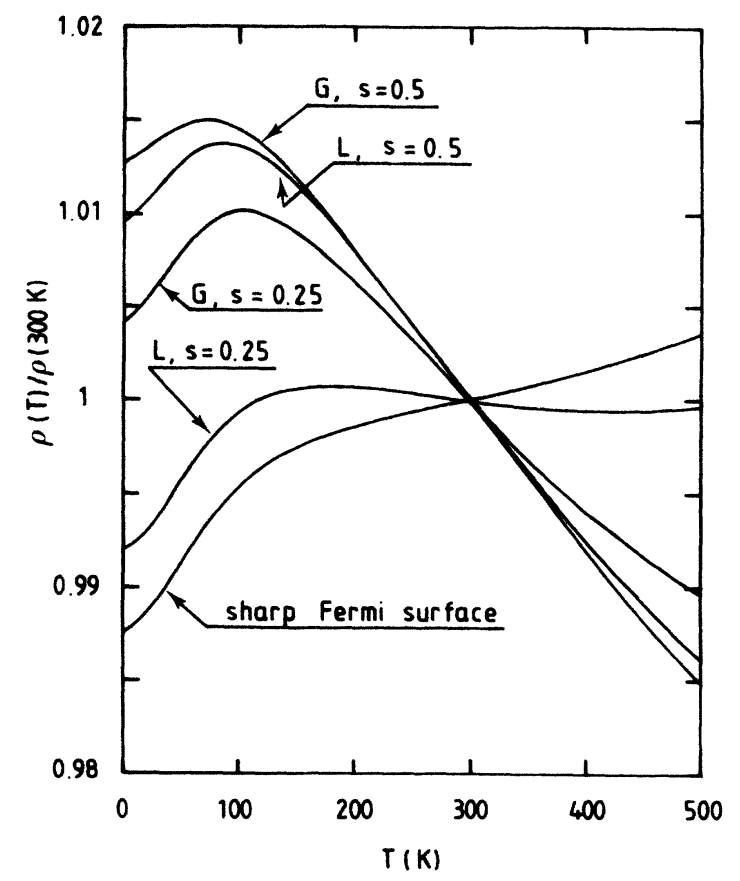

FIG. 4. Resistivity of $\mathrm{Ca}_{30} \mathrm{Al}_{70}$ metallic glass as a function of temperature. The curves are computed for a sharp Fermi surface and for Gaussian (G) and Lorentzian (L) wave packets. The corresponding value of the width parameter $s$ is indicated. In all cases, $k_{F}=1.18 \AA^{-1}$. 


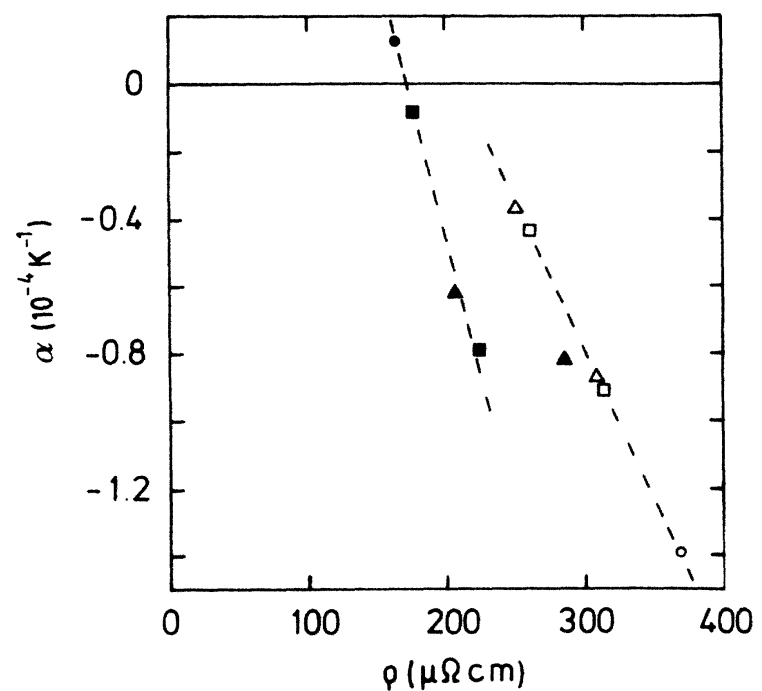

FIG. 5. Temperature coefficient of resistivity as a function of resistivity. Open symbols refer to $\mathrm{Ca}_{60} \mathrm{Al}_{40}$ and solid to $\mathrm{Ca}_{30} \mathrm{Al}_{70}$. The circles give the sharp-Fermi-surface values and the triangles and squares show the results of using Gaussian and Lorentzian wave packets, respectively. The widths of the wave packets are the same as those in Figs. 4 and 5.

curve does not change. The direction of change in the resistivity with increasing wave-packet width depends on the composition.

For a more detailed comparison with experiments, the computed temperature dependence of resistivity is shown in Fig. 6 for Ca-Al compounds containing 20, 40, 45, and 60 at. $\%$ Al. All these calculations are due for a sharp Fermi surface-according to the preceding discussions the inclusion of the Fermi-surface blurring only slightly increases the room-temperature TCR [below (see Fig. 9), it is shown that for this concentration range a nonzero wave-packet width decreases the resistivity]. There are experimental measurements ${ }^{5,20}$ for concentrations $20-45$ at. \% Al. These compare favorably with the theoretical results: In each case the temperature dependence above 50 $K$ is about linear with a negative TCR. The dependence is found to be weakest for the 20-at. \% Al curve and to become steeper when going towards 45 at. $\%$ with an obvious saturation around 45 at. $\% \mathrm{Al}$. For a compound of 60 at. \% Al our calculations show still the same kind of temperature dependence, with a slope about halfway between the curves corresponding to 20 and 40 at. $\% \mathrm{Al}$.

The theoretical and experimental TCR's as a function of composition at $300 \mathrm{~K}$ are shown in Fig. 7. The calculated and measured curves indicate the same qualitative features. Moreover, the TCR clearly shows how the computed and measured TCR's agree even quantitatively for 20 at. \% Al, but the experimental TCR decreases far more rapidly than the theoretical one when going towards higher $\mathrm{Al}$ contents. The theoretical curve has a minimum at 45 at. \% Al. To test this there are unfortunately no measurements for compositions with more than 45 at. \% Al.

Mizutani and Matsuda ${ }^{20}$ have measured a prominent, linear dependence between the TCR and the resistivity,

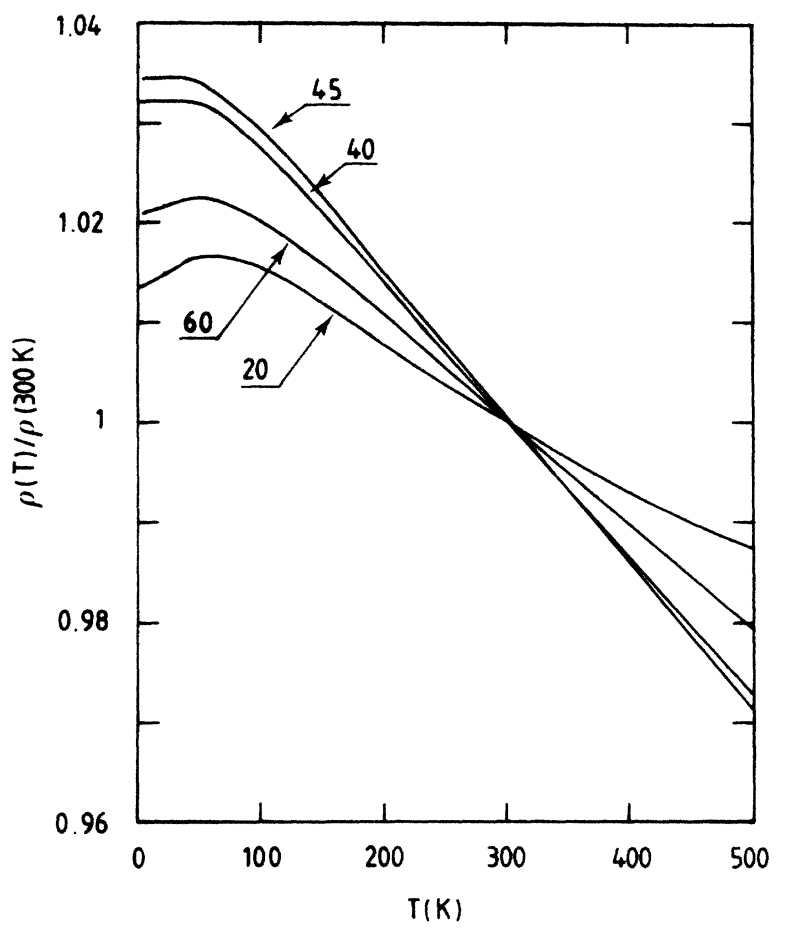

FIG. 6. Temperature dependence of resistivity for different $\mathrm{Ca}-\mathrm{Al}$ metallic glasses. The numbers give the $\mathrm{Al}$ content in at. \%. A sharp Fermi surface was used and $k_{F}=1.18 \AA^{-1}$ for all cases.

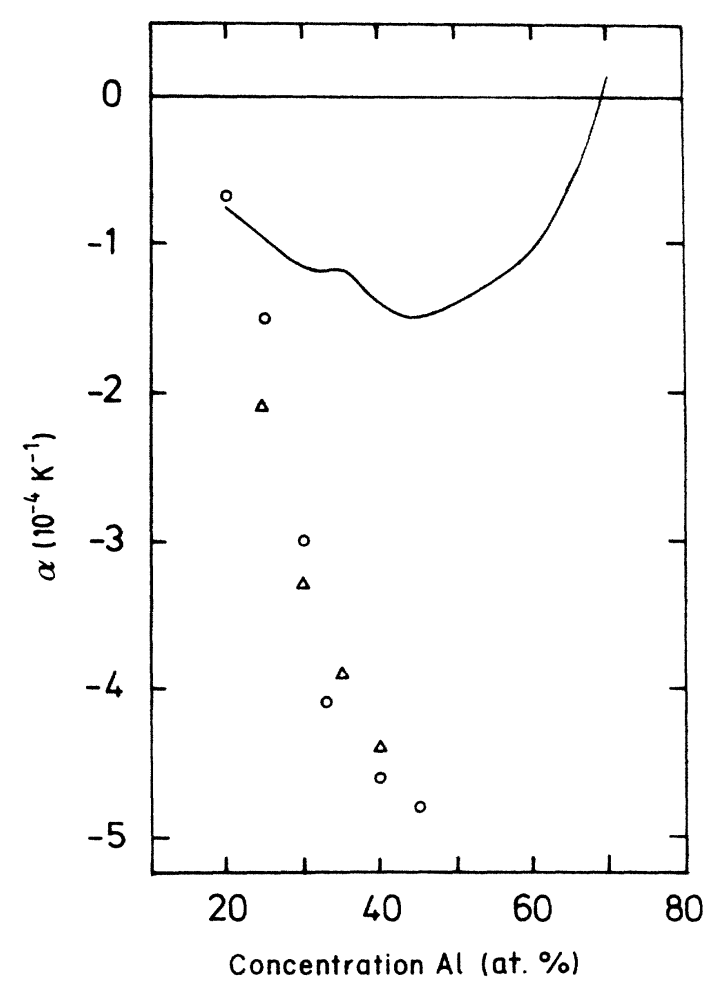

FIG. 7. Temperature coefficient of resistivity (TCR) as a function of composition at room temperature. Present results are shown by solid line, circles refer to the experimental measurements by Love et al. (Ref. 5), and triangles to those by Mizutani and Matsuda (Ref. 20). 
(i.e., the Mooij correlation) at $300 \mathrm{~K}$ for $\mathrm{Ca}-\mathrm{Al}$ compounds with $25-40$ at. $\% \mathrm{Al}$. The corresponding theoretical results for compositions with $20-70$ at. $\% \mathrm{Al}$ are shown in Fig. 8. One can see a clear correlation between the TCR and the resistivity: The TCR decreases as the resistivity increases, as is also confirmed experimentally. The theoretical values are, however, more scattered, and in the high-resistivity end the decrease in the TCR seems to set in more slowly than the linear dependence would lead one to expect.

Figure 9 shows the composition dependence of resistivity at room temperature. Four theoretical curves are drawn, corresponding to different assumptions about Fermi-surface blurring and the value of $k_{F}$. Also, the available experimental results ${ }^{5,20}$ are shown. There is a rather good agreement between experiment and theory for curves with $k_{F}=1.18 \AA^{-1}$. The assumption of a sharp Fermi surface is seen to best reproduce the experimentally found fast and large increase in the resistivity with an apparent maximum around 45 at. \% Al. This same kind of behavior also results when the blurring of the Fermi surface is taken into account, but the rise in the resistivity and the position of the maximum deviate more from the experiments the larger the width of the wave packet is (note that even for the same $\Delta k$ the FWHM is larger for the Gaussian- than the Lorentzian-type wave packet). These results, along with the temperature-dependence curves (Figs. 3, 4, and 7), indicate that the width of the wave packet to be used in the theory developed here is actually very small; a "correct" value for $s$ is probably closer to 0 than 0.5 .

The use of partial structure factors enables one to express the resistivity in the form [see Eq. (12)]

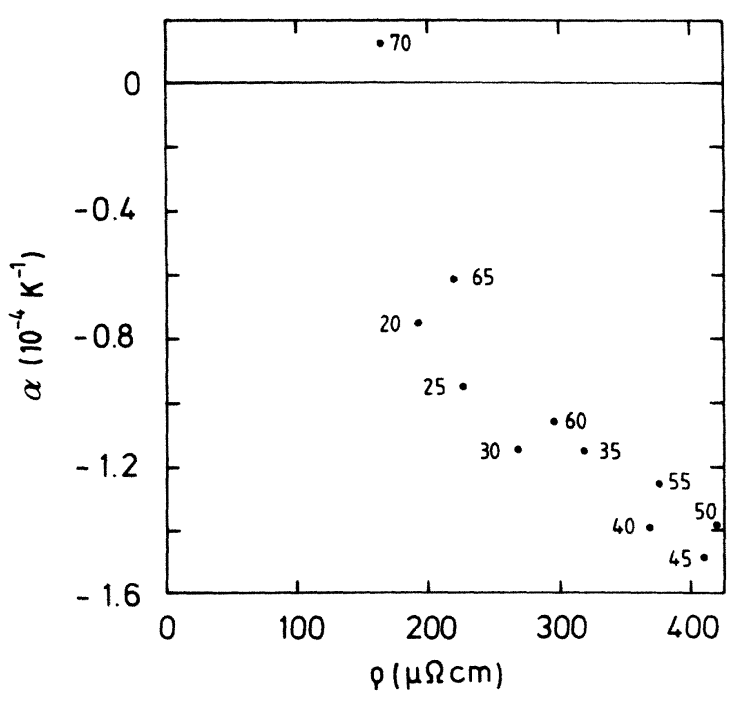

FIG. 8. Relation between TCR and the resistivity at $300 \mathrm{~K}$ for $\mathrm{Ca}-\mathrm{Al}$ metallic glass. Each point corresponds to a different composition. This is indicated by the number giving the $\mathrm{Al}$ content in at. \%. The sharp-Fermi-surface formulation has been used.

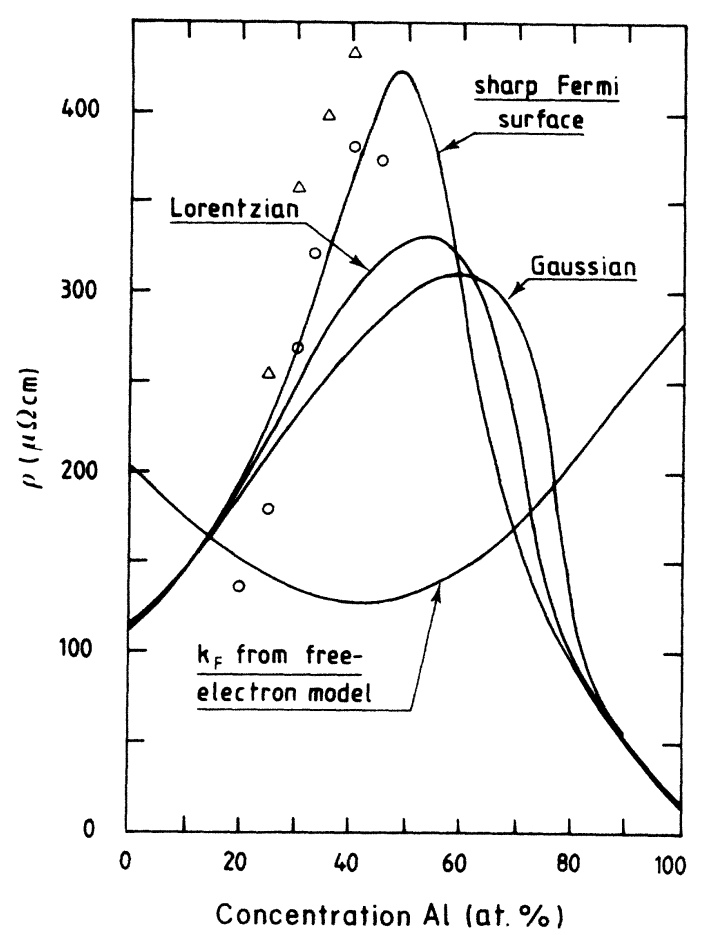

FIG. 9. Resistivity of Ca-Al metallic glass as a function of composition. Two curves have been calculated by taking into account the blurring of Fermi surface; these are marked as "Lorentzian" and "Gaussian" according to the type of the wave packet used. The wave-packet-width parameter has a value $s=0.5$ in both cases. One of the sharp-Fermi-surface curves was calculated by using the Fermi-wave-vector value from the free-electron formula; in all the other cases $k_{F}=1.18$ $\AA^{-1}=$ const for whole concentration range. The calculations correspond to $T=300 \mathrm{~K}$. The experimental points shown by the circles $(T=295 \mathrm{~K})$ are from Love et al. (Ref. 5) and the triangles $(T=300 \mathrm{~K})$ refer to the measurements by Mizutani and Matsuda (Ref. 20).

$$
\rho=\rho_{\mathrm{Al}-\mathrm{Al}}+\rho_{\mathrm{Ca}-\mathrm{Ca}}+\rho_{\mathrm{Ca}-\mathrm{Al}},
$$

where $\rho_{i-j}$ is the term including the partial structure factor $S_{i j}(k, \omega)$. Then one may roughly say that $\rho_{i-i}$ describes the resistivity due to scattering from atoms of type $i$ and $\rho_{i-j}(i \neq j)$ is a kind of interference term. A plot of these components as a function of composition is shown in Fig. 10. The two sets of curves correspond to a sharp Fermi surface and a Gaussian wave packet with $s=0.5$. Figure 10 reveals various interesting features. First, in $\mathrm{Ca}-\mathrm{Al}$ metallic glass, $\mathrm{Ca}$ is a strong-scattering element giving a large contribution to the total resistivity. For large $\mathrm{Al}$ concentrations, $\rho_{\mathrm{Al}-\mathrm{Al}}$ turns out to be small, but for intermediate concentrations $\rho_{\mathrm{Al}-\mathrm{Al}}$ rises unexpectedly high, above $100 \mu \Omega \mathrm{cm}$. This effect is obviously a consequence of changes in atomic [ $\left.S_{\mathrm{Al}-\mathrm{Al}}(k, \omega)\right]$ and electronic (value of $k_{F}$ ) structure. When approaching the pure-metal ends, the interference term $\rho_{\mathrm{Ca}-\mathrm{Al}}$ goes to zero, but between these two limits $\rho_{\mathrm{Ca}-\mathrm{Al}}$ is seen to provide a large fraction of the total resistivity. At high $\mathrm{Al}$ concentrations, $\rho_{\mathrm{Ca}-\mathrm{Al}}$ is even negative. This is a structural effect following from 


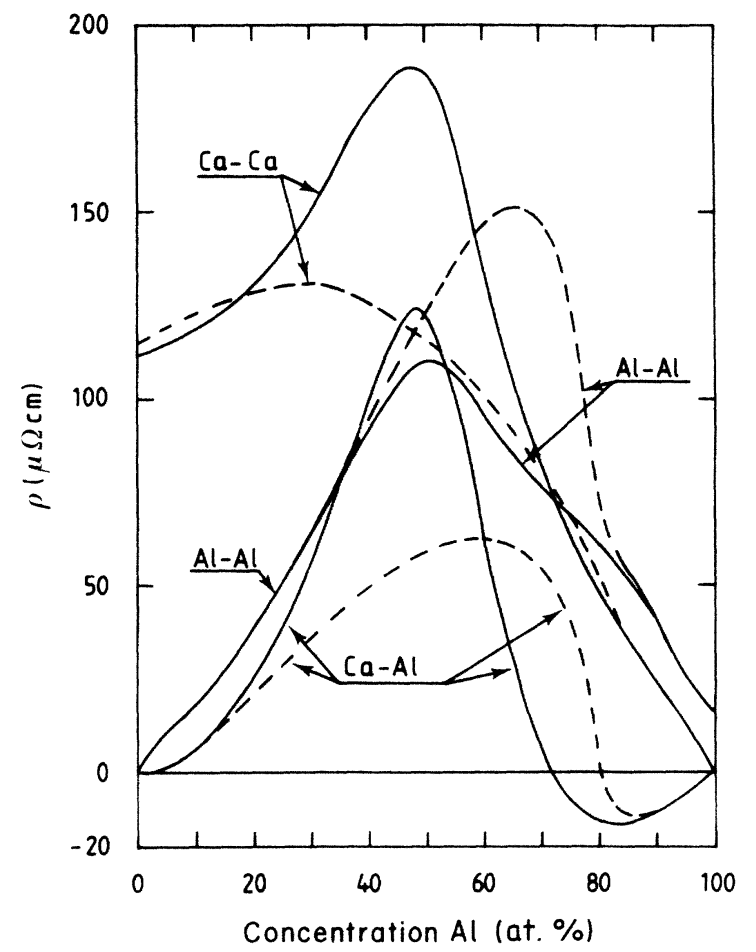

FIG. 10. Composition dependences of the three resistivity terms $\rho_{\mathrm{Al}-\mathrm{Al}}, \rho_{\mathrm{Ca}-\mathrm{Ca}}$, and $\rho_{\mathrm{Ca}-\mathrm{Al}}$ which add up to the total resistivity. The solid curves have been calculated using a sharp Fermi surface and the dashed ones are from the wave-packet formulation with a Gaussian wave packet $(s=0.5)$. The temperature is $T=300 \mathrm{~K}$ and $k_{F}=1.18 \AA^{-1}$.

$S_{\mathrm{Ca}-\mathrm{Al}}(k, \omega)$ not being positive definite. As noticed earlier, the wave-packet formulation is not seen to give any new qualitative features, but quantitative changes are notable, especially at intermediate concentrations. An interesting property evident in Figs. 9 and 10 is that when approaching the pure-metal ends, the resistivity becomes essentially independent of the wave-packet width; only for the highresistivity $\mathrm{Ca}$ can a small difference be seen.

The $k_{F}$ dependence of the resistivity is presented in Fig. 11 , where the resistivities of $\mathrm{Ca}_{60} \mathrm{Al}_{40}$ and $\mathrm{Ca}_{30} \mathrm{Al}_{70}$ compounds are plotted as a function of the Fermi wave vector. The fast increase for small $k_{F}$ and the approach towards zero at large $k_{F}$ result from the $1 / n_{e}^{2}$ factor in Eq. (12). The important point in Fig. 11 is the sharp peak with a very large increase in resistivity in the vicinity of $k=1.2$ $\AA^{-1}$. The curves concretely show the sensitive dependence of resistivity on the value of $k_{F}$, i.e., on the electronic structure of the metallic glass. The fact that not only the height but also the positions of the peaks of Fig. 11 change with composition makes the concentration dependence of $k_{F}$ of utmost importance in the diffraction model. The results of the calculations for the concentration dependence of resistivity (Fig. 9) clearly support the use of $k_{F} \cong 1.18 \AA^{-1} \simeq$ const for the whole composition range, as deduced from Hall-effect measurements. ${ }^{19}$ The application of the free-electron formula for $k_{F}$, on the other hand, is seen to result in an incorrect composition dependence of resistivity (see Fig. 9). This finding is con-

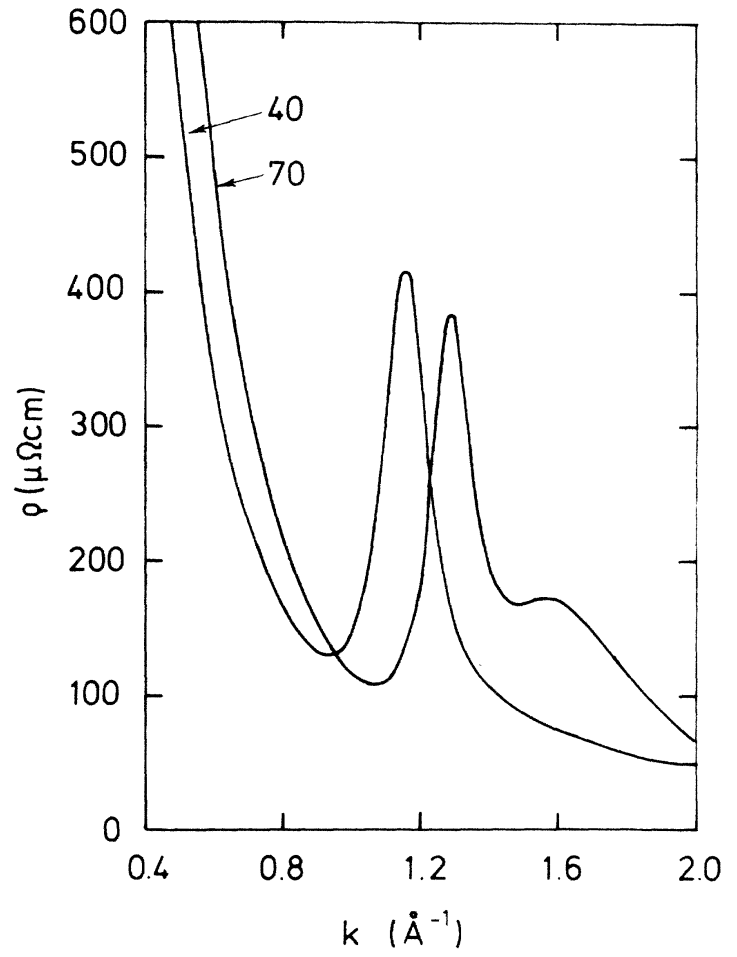

FIG. 11. Resistivity as a function of the Fermi wave vector at $T=300 \mathrm{~K}$. Two alloys containing 40 and 70 at. $\% \mathrm{Al}$ are considered. The sharp-Fermi-surface formulation is used.

sistent with the conclusion ${ }^{20,23}$ that $\mathrm{Ca}-\mathrm{Al}$ metallic glass is not a free-electron-like system.

We also computed the resistivity using a pseudopotential instead of a $t$ matrix (the results to be described are for the sharp-Fermi-surface formulation with $k_{F}=1.18$ $\AA^{-1}$ throughout). The Ashcroft model potential ${ }^{9}$ and the $a b$ initio pseudopotential by Bachelet, Hamann, and Schlüter ${ }^{10}$ were applied. In the case of Ashcroft potential, the room-temperature resistivity of $\mathrm{Ca}_{60} \mathrm{Al}_{40}$ was 80 $\mu \Omega \mathrm{cm}$ and $\rho(T=0)$ was smaller than $\rho(T=300 \mathrm{~K})$ with the $\rho(T)$ curve turning downward around $300 \mathrm{~K}$. All this is clearly in contradiction to the experiments. The $a b$ initio pseudopotential gave distinctly better results: Now $\rho(T=300 \mathrm{~K}) \simeq 170 \mu \Omega \mathrm{cm}$ and the temperature dependence was closer to the correct form with $\rho(T=0)$ $>\rho(T=300 \mathrm{~K})$, but the dependence was still markedly less steep than that shown in Fig. 3. Both potentials yielded a composition dependence of $\rho(c)$ being convex upward with a maximum at $c \simeq 40-50$ at. $\%$ Al. These findings suggest that the $t$-matrix formulation of scattering is essential for high-resistivity metallic glasses formed of simple metals, even if good results have been obtained with pseudopotentials for the usual, low-resistivity glasses of simple metals.

Finally, we comment on the effect of the Pippard function $^{13}$ and the Sham-Ziman approximation. ${ }^{14}$ For the inclusion of electron mean-free-path effects on the electron-phonon. interaction in the form of the Pippard 
function, we found what Meisel and Cote did: ${ }^{24}$ The room-temperature values of resistivity and the TCR decrease and the low-temperature minimum of the $\rho(T)$ curve become shallower. The absolute value of resistivity decreased only $1-2 \%$, but the change in temperature dependence was notable and a reduction by roughly a factor of 2 in the TCR was seen. The Sham-Ziman approximation is meant to take into account the neglected terms in the dynamical structure factor when the multiphonon expansion is truncated at the one-phonon term. Correspondingly, the Sham-Ziman approximation is expected to have some effect only at high temperatures by increasing the resistivity. At low temperatures multiphonon processes are scarce in any case; thus, as a result, multiphonon terms increase the TCR. The calculation confirmed this: Below $100 \mathrm{~K}$ practically no change in resistivity was seen; at $300 \mathrm{~K}$ it increased by about $0.7 \%$ and at $500 \mathrm{~K}$ by about $1.7 \%$.

\section{DISCUSSIONS AND CONCLUSIONS}

The results given show that the calculations reproduce well, both qualitatively and quantitatively, the magnitude and the composition dependence of the resistivity. For the temperature dependence the agreement is only qualitative; experimentally, considerably steeper temperature dependences have been found. The same observation was made earlier for the $\mathrm{Mg}-\mathrm{Zn}$ system in paper I. Very recently, Hafner ${ }^{25}$ considered this seeming inadequacy of the diffraction model. He points out that usually, as in this paper, the thermal expansion of the material is not taken into account. For crystalline metals this is justified, since the change in $\rho(T)$ from $T=0$ to room temperature is very large, but in the case of metallic glasses this change is only a few percent and then even the small effect of thermal expansion should be accounted for. Now the thermal expansion would affect $n_{i}$ and the $S_{a b}(q, \omega)$ 's in Eq. (12). For the dynamical structure factors the resulting change in $\rho$ is difficult to estimate, but a decrease in $n_{i}$ due to expansion will directly decrease $\rho$ also, when temperature increases. This makes the TCR smaller: Hafner found a decrease in the TCR by a factor of 2-3 due to thermal expansion. Depending on the composition, this is just what is needed in Fig. 7. From Fig. 8 it is obvious that in order to make the $\alpha$-versus- $\rho$ dependence linear, the volume effect, i.e., the expansion, should be largest for those compositions with the largest resistivities. It would thus be of interest to know the thermal expansion of $\mathrm{Ca}-\mathrm{Al}$ metallic glass as a function of composition.

The calculated composition dependence of resistivity (Fig. 9) agrees rather well with experiment. Since the phase shifts used were independent of concentration, it appears that the concentration dependence of resistivity is mostly due to that of dynamical structure factors and the Fermi wave vector. The determination of the phase shifts from the atomic-type potential excludes composition effects such as charge transfer. This procedure can be expected to be most accurate near the ends of the concentration range and least accurate for intermediate compositions. However, from Fig. 9 it can be seen that the agree- ment with experiment is best at intermediate concentrations and seems to start to degrade when approaching the pure-Ca end. Then the deviation close to pure $\mathrm{Ca}$ hints of a possible inadequacy of the $S_{a b}(k, \omega)$ to describe this concentration range, since here the phase shifts should be best. Here the composition dependence of $S_{a b}(k, \omega)$ follows from that of $S_{a b}(k)$, as described in paper I. The static structure factors are obtained from the PercusYevick approximation for hard spheres. ${ }^{26}$ In general, this gives a good account of the composition dependence, but it would be useful to have $S_{a b}(k)$ for amorphous alloys (especially for small concentrations of the other constituent) obtained using other methods.

The results indicate that it is not vital to include the Fermi-surface blurring in the theory. It is true that the blurring changes somewhat the absolute value of resistivity as well as its temperature and composition dependence, but the changes are only slight modifications to the shape-Fermi-surface results and no new features emerge. The resistivity components $\rho_{\mathrm{Al}-\mathrm{Al}}, \rho_{\mathrm{Ca}-\mathrm{Ca}}$, and $\rho_{\mathrm{Ca}-\mathrm{Al}}$ in Fig. 10 show a larger effect than the total resistivity when the wave-packet formulation is used. Specifically, $\rho_{\mathrm{Al}-\mathrm{Al}}$ increases and $\rho_{\mathrm{Ca}-\mathrm{Ca}}$ predominantly decreases when the blurring is included. The changes are largest for intermediate concentrations. A possible explanation is that the propagation of the wave packet is distorted most in those cases where, in addition to the structural disorder, the chemical disorder as well is large.

Various authors ${ }^{24,25,27}$ have concluded that the diffraction model is well suited to describe the low-resistivity metallic glasses composed of simple metals, but questions have been raised as to whether the model can account for high-resistivity systems. To the authors' knowledge the present calculation is the first in which the model is applied to a high-resistivity simple-metal amorphous alloy. The results obtained compare favorably with the experimental findings: The magnitudes of the resistivity and concentration dependences are all reproduced by the model. Furthermore, we find the same interrelationship between the resistivity and its temperature coefficient (the Mooij correlation) as is evident from experimental measurements. This clearly supports the application of the diffraction model to high-resistivity systems as well-at least in the cases where the alloy constituents are simple metals.

A new method was proposed to take into account the scattering-induced blurring of the Fermi surface. When electrons are considered as wave packets, the uncertainty in the electron momentum enters naturally as the width of the wave packet. This was clearly seen to affect the resistivity, but precise quantitative statements are difficult to make since there is no way of unambiguously determining the width of the wave packet. However, the changes are not expected to be extensive, and considering all the other parameters of the model, the usual sharp-Fermi-surface formulation is sufficient in most cases. The largest effects were seen at intermediate concentrations, i.e., for compositions where there is also a considerable chemical disorder in addition to the structural one.

By far, the most important single parameter of the model was seen to be the Fermi wave vector $k_{F}$. Here, its 
value was deduced from the Hall-effect measurements showing $k_{F} \simeq$ const $\simeq 1.18 \AA^{-1}$ for the whole concentration range. This yielded good results for the resistivity. The use of the free-electron formula for $k_{F}$, on the other hand, results in a very poor composition and temperature dependence of the resistivity. In this context it is appropriate to stress the need to carefully determine the value of $k_{F}$ when applying the diffraction model. For low-resistivity simple metals the free-electron formula seems to work well, but in the high-resistivity case scaling with experimental Hall-effect measurements is in order.

The concentration dependence of the resistivity was seen to be determined mostly by that of $k_{F}$ and $S_{a b}(k, \omega)$. This calls for the use of realistic dynamic structure factors when reliable estimates of resistivity are wanted. The decomposition of total resistivity into components due to scattering from $\mathrm{Al}$ and $\mathrm{Ca}$ atoms, and an interference term, revealed their markedly different concentration dependencies. It is thus essential to maintain the full theory with partial structure factors in order to account for the sensitive interplay between different terms.

Much of the high resistivity in Ca-Al metallic glass alloys was seen to follow from electron scattering from $\mathrm{Ca}$ atoms. For intermediate concentrations, however, very sizable portions also resulted from $\mathrm{Al}$ scattering and the interference term.

The calculated temperature dependence of resistivity turned out to be smaller than the experimental one. The diffraction model, even with mean-free-path corrections, thus seems unable to account for the large negative TCR's observed in high-resistivity alloys. As shown elsewhere, ${ }^{25}$ a possible reason for this is the disregard of thermal expansion in the calculations. This explanation cannot, unfortunately, be tested, since the necessary thermodynamic data are not available.

\section{ACKNOWLEDGMENT}

We thank Dr. M. Puska for providing the computer programs used to compute the scattering phase shifts.

\section{APPENDIX: DIFFERENTIAL CROSS SECTION FOR A WIDE WAVE PACKET}

The following formulation is based on the derivation of a differential cross section for the usual case of a peaked wave packet found in standard quantum-mechanics text books. $^{7}$ After scattering, the wave packet of Eq. (2) is, at $r$ at time $t$,

$$
\psi(\mathbf{r}, t)=\psi\left(\mathbf{r}-\mathbf{v}_{0} t, 0\right) e^{i \omega_{0} t}+\frac{e^{i \omega_{0} t}}{r(2 \pi)^{3 / 2}} \int d^{3} k \varphi(\mathbf{k}) f_{\mathbf{k}}(\hat{\mathbf{r}}) e^{i\left|k r-\mathbf{k} \cdot\left(\mathrm{r}_{0}+\mathrm{v}_{0} t\right)\right|},
$$

where $\mathbf{v}_{0}=\hbar \mathbf{k}_{0} / m$ is the initial velocity of the particle and $\hbar \omega_{0}=\frac{1}{2} m v_{0}^{2}$. Note that in (A1) the scattering amplitude $f_{\mathbf{k}}(\hat{\mathbf{r}})$ has been kept inside the integral. The total number of particles scattered into solid angle $d \Omega$ at $\mathbf{r}$ is

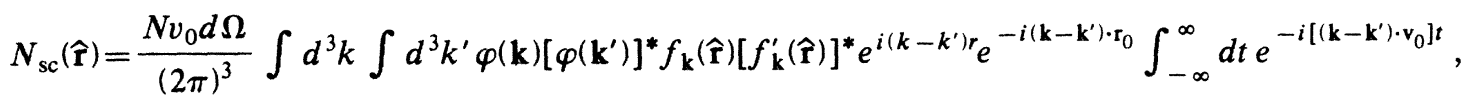

where $N$ is the total number of incident particles. The $t$ integral above gives a $\delta$ function:

$$
2 \pi \delta\left|\left(\mathbf{k}-\mathbf{k}^{\prime}\right) \cdot \mathbf{v}_{0}\right|=\frac{2 \pi}{v_{0}} \delta\left(\left|\mathbf{k}-\mathbf{k}^{\prime}\right| \cos \theta\right),
$$

where $\theta$ is the angle between $\mathbf{v}_{0}$ and $\mathbf{k}-\mathbf{k}^{\prime}$. This means that in (A2) either $\mathbf{k}=\mathbf{k}$ or $\mathbf{v}_{0} \perp(\mathbf{k}-\mathbf{k})$. In the latter case, because of the term $e^{i\left(k-k^{\prime}\right) r_{;}}$, the largest contribution is obtained when $k \approx k^{\prime}$, especially when $k \approx k_{0}$ and $k^{\prime} \approx k_{0}$ [since $\varphi(k)$ has maximum at $k=k_{0}$ ]. Then, due to the constraint $\mathbf{v}_{0} \perp(\mathbf{k}-\mathbf{k})$, we have $\cos \left(\mathbf{v}_{0}, \mathbf{k}\right) \approx \cos \left(\mathbf{v}_{0}, \mathbf{k}^{\prime}\right)$, and since $\varphi(\mathbf{k})$ and $f_{\mathbf{k}}(\hat{r})$ have azimuthal symmetry around $\mathbf{v}_{0}$, one may rotate $\mathbf{k}^{\prime}$ to get $\mathbf{k} \approx \mathbf{k}^{\prime}$. This shows that in $\mathrm{Eq}$. (A2) one may safely substitute, for the $\delta$ function of (A3),

$$
2 \pi \delta\left[\left(\mathbf{k}-\mathbf{k}^{\prime}\right) \cdot \mathbf{v}_{0}\right] \rightarrow \frac{C}{v_{0}} \delta\left(\mathbf{k}-\mathbf{k}^{\prime}\right)
$$

where $C$ is a normalization constant. Now we obtain, for the number of scattered particles,

$$
N_{\mathrm{sc}}(\hat{\mathbf{r}})=\frac{N d \Omega}{(2 \pi)^{3}} C \int d^{3} k|\varphi(\mathbf{k})|^{2}\left|f_{\mathbf{k}}(\hat{\mathbf{r}})\right|^{2} .
$$

Using the same reasoning the number of incident particles can be expressed as

$$
N_{\text {inc }}=\frac{N}{(2 \pi)^{3}} C \int d^{3} k|\varphi(\mathbf{k})|^{2} .
$$

The differential cross section for a wide wave packet is then

$$
\frac{d \sigma}{d \Omega}=\frac{\int d^{3} k|\varphi(\mathbf{k})|^{2}\left|f_{\mathbf{k}}(\boldsymbol{r})\right|^{2}}{\int d^{3} k|\varphi(\mathbf{k})|^{2}}
$$

*Also at Department of Physics, University of Jyväskylä, SF40100 Jyväskylä 10, Finland.

1J. Laakkonen and R. M. Nieminen, J. Phys. F 13, 2265 (1983).

2P. J. Cote and L. V. Meisel, Phys. Rev. Lett. 40, 1586 (1978).

${ }^{3}$ L. V. Meisel and P. J. Cote, Phys. Rev. B 30, 1743 (1984).
${ }^{4}$ A. Ferraz and N. H. March, Phys. Chem. Liq. 8, 271 (1979).

${ }^{5}$ D. P. Love, F.-C. Wang, D. G. Naugle, C. L. Tsai, B. C. Giessen, and T. O. Callaway, Phys. Lett. 90A, 303 (1982). 6J. M. Ziman, Philos. Mag. 6, 1013 (1961).

7See, e.g., E. Merzbacher, Quantum Mechanics (Wiley, New 
York, 1970).

${ }^{8}$ F. Herman and S. Skillman, Atomic Structure Calculations (Prentice-Hall, New Jersey, 1963). Perdew-Zunger parametrization [J. P. Perdew and A. Zunger, Phys. Rev. B 23, 5048 (1981)] of the Ceperley-Alder exchange-correlation energy functional [D. M. Ceperley and B. J. Alder, Phys. Rev. Lett. $45,566(1980)]$ is used in the local-density approximation.

${ }^{9}$ N. W. Ashcroft, Phys. Lett. 23, 48 (1966).

${ }^{10} \mathrm{G}$. B. Bachelet, D. R. Hamann, and M. Schlüter, Phys. Rev. B 26, 4199 (1982).

${ }^{11}$ N. W. Ashcroft and J. Lekner, Phys. Rev. 145, 83 (1966).

12R. Taylor, J. Phys. F 8, 1699 (1978).

${ }^{13}$ A. B. Pippard, Philos. Mag. 46, 1104 (1955); J. M. Ziman, Electrons and Phonons (Clarendon, Oxford, 1960), Chap. V.

${ }^{14}$ L. J. Sham and J. M. Ziman, in Solid State Physics, edited by F. Seitz and D. Turnbull (Academic, New York, 1963), Vol. 15.

15J. Hafner, Phys. Rev. B 21, 406 (1980).

${ }^{16} \mathrm{G}$. Leibfried and N. Breuer, Point Defects in Metals I, Vol. 81 of Springer Tracts in Modern Physics, edited by G. Höhler and E. A. Niekisch (Springer, Berlin, 1978).
${ }^{17}$ K. A. Gschneidner, in Solid State Physics, edited by F. Seitz and D. Turnbull (Academic, New York, 1964), Vol. 16, p. 275.

${ }^{18}$ F. Cyrot-Lackmann, Phys. Rev. B 22, 2744 (1980).

${ }^{19}$ C. L. Tsai, J. Hong, and B. C. Giessen, in Proceedings of the Fourth International Conference on Rapidly Quenched Metals, edited by T. Masumoto and K. Suzuki (Japan Institute of Metals, Sendai, 1981), Vol. 2, p. 1327.

${ }^{20}$ U. Mizutani and T. Matsuda, J. Phys. F 13, 2115 (1983).

${ }^{21}$ S. R. Nagel, U. M. Gubler, C. F. Hague, J. Krieg, R. Lapka, P. Oelhafen, H.-J. Güntherodt, J. Evers, A. Weiss, V. L. Moruzzi, and A. R. Williams, Phys. Rev. Lett. 49, 575 (1982).

22J. H. Mooij, Phys. Status Solidi A 17, 521 (1973).

${ }^{23}$ W. A. Hines, P. Miller, A. Paoluzi, C. L. Tsai, and B. C. Giessen, J. Appl. Phys. 53, 7789 (1982).

${ }^{24}$ L. V. Meisel and P. J. Cote, Phys. Rev. B 27, 4617 (1983).

25J. Hafner, J. Non-Cryst. Solids 69, 325 (1985).

${ }^{26}$ N. W. Ashcroft and D. C. Langreth, Phys. Rev. 156, 685 (1967); N. W. Ashcroft and D. C. Langreth, ibid. 159, 500 (1967).

${ }^{27}$ U. Mizutani and T. Yoshida, J. Phys. F 12, 2331 (1982). 